\title{
Lupus Nephritis: A comprehensive review
}

\author{
Sigdel M R, Shah D S, Raut K B \\ Department of Nephrology, TU Teaching Hospital, Kathmandu, Nepal
}

Correspondence address: Mahesh Raj Sigdel, Department of Nephrology, TU Teaching Hospital, Kathmandu, Nepal

Email: maheshsigdel@hotmail.com

\section{Introduction and epidemiology}

The term 'lupus' (Latin, 'wolf') was first used during the Middle Ages to describe erosive skin lesions. Systemic lupus erythematosus (SLE) is a complex, chronic multi system autoimmune connective tissue disease that is characterized by protean array of clinical manifestations and production of autoantibodies to a host of endogenous nuclear antigens.1-3. Involvement of kidney in SLE is known as lupus nephritis (LN). LN is the most common severe manifestation of SLE with increased risk of death and endstage renal disease (ESRD)., ${ }^{1,4}$

The incidence and prevalence of lupus and LN are influenced by age, gender, ethnicity, and geographic region.4 The overall incidence ranges from 1.8 to 7.6 cases per 100,000 people. ${ }^{1,9}$ The prevalence ranges from approximately 40 cases per 100,000 persons among Northern Europeans to more than 200 per 100,000 persons among blacks; the highest prevalence is reported in Brazil. SLE predominantly affects young women of reproductive age, peak incidence is at 15 to 45 years. Female to male ratio is 10:1. This gender predominance is less pronounced in children and older individuals. ${ }^{1,4,10,11}$ Nearly 35 to $50 \%$ patients of SLE have clinical renal disease at presentation; it reaches to $>60 \%$ during follow up. The prevalence of nephritis is significantly higher in African Americans, Afro-Caribbeans, Asians and Hispanics than in white Caucasians. ${ }^{1,4,6,12}$

\section{Etiology and pathogenesis}

Healthy people get rid of the autoantigen-specific B cells and $\mathrm{T}$ cells through different mechanisms - deletion, anergy or receptor editing.5 Autoimmune disease occurs when the adaptive immune response is mounted against 'self '-antigens, which cannot be removed by effector mechanisms ie loss self tolerance.13 Certain genetic, hormonal, and environmental factors influence the development, course and severity of lupus. ${ }^{1,4,5,14}$ The pathogenesis of SLE can be summarized as: ${ }^{1,4,5,13-15}$

- Background predisposition is conferred by genetic factors; hormonal factors play some role.

- Environmental factors provoke excessive apoptosis. During apoptosis, several proteins and nucleosomes are released from host cells and undergo alteration to form autoantigens which are clustered in blebs at the surface of apoptotic cells. ${ }^{15}$

- Defective clearance of apoptotic cells by phagocytes.

- Apoptotic blebs with modified autoantigens are recognized by Toll-like receptors (TLRs) of dendritic cells (DCs), the professional antigen-presenting cells (APCs) and then are phagocytosed by them.

- Ingestion of apoptotic blebs leads to maturation of the DCs with enhanced expression of the activation markers and co-stimulatory molecules CD86 and CD40; they then present these modified autoantigens to $\mathrm{T}$ cells in an immunogenic way. ${ }^{15}$

- Each T cell carries a surface-receptor for antigen complexed with MHC molecule on the surface of an APC, "signal 1". To stimulate the T cell, APC must also make a second molecular interaction with the $\mathrm{T}$ lymphocyte through costimulation, "signal 2", mediated by interaction costimulatory pairs; CD80 (B7.1) and CD86 (B7.2) on APCs with specific T cell receptors (CD28 and its homolog, cytotoxic T lymphocyte-associated antigen 4 [CTLA4 ie CD152]). CD28 provides stimulatory signal; CTLA4 delivers signals that attenuate $\mathrm{T}$ cell proliferation.5 Activation of Signals 1 and 2 leads to expression of cytokines, especially interleukin-2. These cytokines activate the mTOR via the janus kinase 3 and phosphoinositide-3 kinase signal transduction pathways, "Signal 3", leading to further propagation of the lymphocyte cell cycle. $^{5}$

- T-cell cytokines affect B cells by stimulating cell division, switching antibody production from IgM to the more pathogenic and tissue damaging $\mathrm{IgG}$, and promoting a change in the molecular sequence of the 
secreted antibody so that it binds more strongly to the driving antigen. ${ }^{5}$

- These autoantibodies bind to released nuclear antigens and these immune complexes can activate another subset of DCs, the plasmocytoid DCs, which produce high amounts of interferon-alfa and other type I interferons and amplify the autoimmune response. ${ }^{15}$

- Besides, B cells also have roles in antigen presentation to the T cells and secretion of cytokines and chemokines. A vicious cycle sets in.

The autoantibodies variably present in SLE are - anti nuclear antibody (ANA), anti double stranded DNA (antidsDNA), anti Smith, anti histone, anti-Ro, anti-La, anti Nmethyl-D-aspartate receptor (NMDAR), anti red cell, anti platelet, anti $\mathrm{T}$ cell, anti phospholipid, anti $\beta 2$-glycoprotein 1 , anti-RNP, anti-nucleosome, anti- $\alpha$-actinin, anti C1q antibodies etc. ${ }^{5,6,14,16}$ Observations suggest pathogenetic role for these autoantibodies in lupus: $:^{5,13,14}$

- Maternal transfer of autoantibodies in neonatal lupus.

- Animal models of passive transfer of disease through autoantibody inoculation.

- Observation that lupus activity correlates with titers of anti-dsDNA.

- Maternal anti-Ro antibodies cause fetal heart block.

- Anti red cell antibody and antiplatelet antibody cause hemolytic anemia and thrombocytopenia.

- Immune complexes and complements are found in affected tissues. The antibodies linked to lupus nephritis mainly are anti-dsDNA, anti-nucleosome, anti- $\alpha$ actinin $\&$ anti $\mathrm{C} 1 \mathrm{q}$ antibodies. ${ }^{5}$

The mechanism of tissue injury in lupus can be briefed as: ${ }^{5,14}$

- Immune complexes as the central players in the tissue injury in SLE: Immune complexes localize in the target organ. Their localization within the glomerulus is influenced by size, charge, avidity and the clearing ability of the mesangium. , $^{1,5,14,17}$ Immune complexes accumulate in the subendothelial and mesangial areas first, followed by deposition in the basement membrane and subepithelial areas. ${ }^{14,17}$ In general, mesangial and subendothelial immune deposits are derived from deposition of circulating immune complexes, whereas subepithelial complexes are often formed in situ. Once localized, they lead to complement activation, activation of procoagulant factors, leukocyte infiltration with release of proteolytic enzymes, and activation of cytokines associated with cellular proliferation and matrix formation, the result is glomerulonephritis. ${ }^{4,5,17}$

- Local factors also play key role in causing glomerulonephritis.3 Mesangial cells, interstitial cells, and podocytes acquire antigen presenting properties and secrete proinflammatory cytokines when exposed to interferon- $\gamma$. Mesangial cells from lupus-prone mice produce $\alpha$-actinin, MCP1(monocyte chemoattractant protein 1 ; encoded by the CCL2 gene) and RANTES (regulated upon activation, normal T-cell expressed, and secreted; encoded by the CCL5 gene). ${ }^{3}$ Expression of kallikreins in the kidney may be one of the factors regulating susceptibility to $\mathrm{GN} .^{3}$

- The contributing factors for increased vascular events due to atherosclerosis in patients with SLE are: antibodies to lipoproteins, oxidized lipoproteins, hypertension, metabolic syndrome, increased numbers of endothelial cells and endothelial cell injury from immune complexes and inflammatory molecules. . $^{14,18,19}$

\section{Clinical manifestations}

SLE is a chronic disease with diverse clinical presentation, disease course is characterized by episodes of illness (flares) interspersed with episodes of relative quiescence (remissions). ${ }^{1}$ No single clinical or lab feature is diagnostic of SLE. American College of Rheumatology (ACR) developed criteria for its diagnosis, first in 1971, which was subsequently revised in 1982 and $1997 .{ }^{20}$ It consists of eleven features, namely malar rash, discoid rash, photosensitivity, oral ulcers, arthritis, serositis, renal disorder, neurological disorder, haematologic disorder, immunologic disorder and ANA; 20 presence of any four features, at any time in the natural history of disease, makes the diagnosis of SLE with $96 \%$ sensitivity and specificity. ${ }^{1,4}$ This criteria was primarily developed to produce uniformity in clinical studies, however it may also be used in clinical settings, with some caveats: it is not sensitive enough to diagnose early disease, some systems eg mucocutaneous, are overrepresented and all the features are given equal weightage. ${ }^{1,4}$ It is seen that objective evidence of renal disease, discoid rash and cytopenias have more diagnostic value in lupus compared to other criteria. Other features 
of active SLE include malaise, low-grade fever, poor appetite, weight loss, alopecia, Raynaud's phenomenon, myositis, lung involvement, livedo reticularis, pulmonary hypertension, Libman- Sacks endocarditis, mitral valve prolapse, splenomegaly, lymphadenopathy, thrombotic events and pregnancy complications., 1,4,21-24

A number of scoring systems have been developed to follow the activity of an individual patient with SLE. These include the Systemic Lupus Erythematosus Disease Activity Index (SLEDAI), the British Isles Lupus Assessment Group (BILAG), European Consensus Lupus Activity Measure (ECLAM), the Systemic Lupus Activity Measure (SLAM) etc. The Systemic Lupus International Collaborating Clinics/American College of Rheumatology (SLICC/ACR) damage index is designed to ascertain damage (due to disease itself or drug) in SLE. ${ }^{4,9,12,24}$

\section{Case Definition for $\mathrm{LN}$}

Diagnosis of LN is made based on clinical, serological and renal biopsy findings. $4 \mathrm{LN}$ is diagnosed with: ${ }^{12}$

- Persistent proteinuria $>0.5 \mathrm{gm} /$ day.

- $\quad$ Proteinuria $>3+$ by dipstick, and/or cellular casts including red blood cells [RBCs], hemoglobin, granular, tubular, or mixed); "active urinary sediment" ( $>5 \mathrm{RBCs}$ high-power field [hpf], $>5$ white blood cells [WBCs]/hpf in the absence of infection) can substitute cellular casts.

- Renal biopsy showing immune complex-mediated GN compatible with LN.

- Practically, diagnosis of LN made by rheumatologist or nephrologist is considered valid. ${ }^{12}$

Renal involvement in lupus is manifested variably by asymptomatic proteinuria, hypertension, microscopic hematuria, active urinary sediments, frank nephrotic syndrome, nephritic syndrome, rapidly progressive renal failure. $^{4,21}$ Infrequently, it presents with tubulointerstitial disease, renal tubular acidosis, vasculitis like features, thrombotic disorders associated with a secondary antiphospholipid syndrome (APS), and fibrillary GN.4,21,22

Renal biopsy in patients with SLE

The advantages of renal biopsy are: glomerular disease can be classified, disease can be evaluated for activity, chronicity, tubular and vascular changes and additional or alternative causes of renal disease may be identified. ${ }^{12}$

Indications for renal biopsy in patients with SLE are:12

1. Increasing serum creatinine without convincing alternative causes

2. Proteinuria of $\geq 1.0 \mathrm{gm} /$ day.

3. Combinations of the following, findings confirmed in $\geq 2$ tests done within a short period of time $\&$ in absence of alternative causes:

a. Proteinuria $\geq 0.5 \mathrm{gm} /$ day plus hematuria, defined as $\geq 5 \mathrm{RBCs} / \mathrm{hpf}$.

b. Proteinuria $\geq 0.5 \mathrm{gm} /$ day

\section{Classification of lupus nephritis}

The classification of LN is important in patient care and for comparison of outcome results in trials. ${ }^{1,4,17}$ Pirani and Pollak formulated the first World Health Organization (WHO) classification of LN in 1974. It was further modified in 1982 and 1995. 17 The joint working group of International Society of Nephrology (ISN)/Renal Pathology Society (RPS), formulated a revised classification of LN, the ISN/RPS classification of lupus nephritis in 2003, shown in table 1 . Like the preceding classifications, it is also based exclusively on glomerular pathology. ${ }^{17}$ 
Table 1: ISN/RPS 2003 classification of lupus nephritis ${ }^{17}$

Class I Minimal mesangial lupus nephritis

Normal glomeruli by light microscopy (LM), but mesangial immune deposits by electron microscopy (EM) and immunofluorescence (IF).

Class II Mesangial proliferative lupus nephritis

Purely mesangial hypercellularity defined as $\geq 3$ mesangial cells per mesangial area away from vascular pole or mesangial matrix expansion by LM, with mesangial immune deposits.

Class III Focal lupus nephritis

Focal endo- or extracapillary glomerulonephritis involving $<50 \%$ of all glomeruli, typically with focal subendothelial immune deposits, with or without mesangial alterations.

Class III (A) Active/proliferative lesions: focal proliferative LN.

Class III (C) Chronic/ sclerosing inactive lesions with glomerular scars: focal sclerosing LN.

Class III (A/C) Active and chronic lesions: focal proliferative and sclerosing LN.

Class IV Diffuse lupus nephritis

Diffuse endo- or extracapillary glomerulonephritis involving $\geq 50 \%$ of all glomeruli, typically with diffuse subendothelial immune deposits, with or without mesangial alterations.

Class IV-S (A) Diffuse segmental proliferative LN; $\geq 50 \%$ of the involved glomeruli have segmental lesions

Class IV-G (A) Diffuse global proliferative LN; $\geq 50 \%$ of the involved glomeruli have global lesions

Class IV-S (A/C) Diffuse segmental proliferative and sclerosing LN.

Class IV-G (A/C) Diffuse global proliferative and sclerosing LN.

Class IV-S (C) Diffuse segmental sclerosing LN.

Class IV-G (C) Diffuse global sclerosing LN.

Class V Membranous lupus nephritis

Subepithelial immune deposits by LM, IF or EM, with or without mesangial hypercellularity. With well developed lesions, thickening of the glomerular capillary walls and "spike" formation is seen. When class V lupus

nephritis occurs in combination with class III or IV, both are diagnosed.

Class VI Advanced sclerotic lupus nephritis

$\geq 90 \%$ of glomeruli globally sclerosed with no evidence of ongoing active glomerular disease

A diagnosis of combined class III and class V or class IV and class V made only if subepithelial deposits (membranous involvement) cover at least $50 \%$ of the glomerular capillary surface area in at least $50 \%$ of glomeruli by LM or IF. Class IV lesions may have double contours of the GBMs. Some class III and IV biopsies have focal necrotizing and crescentic lesions. 25 Lupus class IV with crescents in $>50 \%$ of the glomeruli are called crescentic GN. Adequate biopsy consists of $\geq 10$ glomeruli; sections should be cut at $3 \mu .^{17}$

Three salient IF findings in LN include: immune deposits in all four compartments of kidney - glomeruli, tubules, interstitium, and blood vessels, immune deposits in all three components of glomerulus - mesangium, subendothelium and subepithelium and full house staining defined as presence of all three immunoglobulins, $\operatorname{IgG}, \operatorname{IgA}$, and $\operatorname{IgM}$, along with the two complement components, C1q \& C3.,4,26 In EM the immune deposits are typically electron dense and granular. Some deposits have fingerprinting pattern. Sometimes tubuloreticular inclusions are seen within dilated cisternae of the endoplasmic reticulum of endothelial cells. ${ }^{1,4,17}$ 
Sometimes, transformation of histologic class from a more benign (ISN class II or class V) to a more active proliferative lesion (class III or class IV) is seen. With successful treatment, patients can transform from a proliferative class (class III or class IV) to a more benign membranous pattern (class V). ${ }^{1,4}$ An extremely uncommon condition is silent lupus nephritis; patients have active proliferative lesions on biopsy but no clinical or urinary sediment changes and normal lupus serologies. These patients will manifest clinical renal involvement shortly into their course. ${ }^{1,5,25}$

\section{Activity and Chronicity}

Austin et al first developed scoring system for activity and chronicity in renal biopsy. Activity implies potentially reversible lesions and chronicity implies potentially irreversible lesions. The National Institutes of Health (NIH) activity and chronicity scores are used as adjunct to ISN/RPS classification. ${ }^{17}$ Six histologic features are included in the activity index (AI) namely endocapillary proliferation, glomerular leukocyte infiltration, wire loop deposits, fibrinoid necrosis and karyorrhexis, cellular crescents, and interstitial inflammation. Each lesion is scored 0 to $3+$, the severe lesions of crescents and fibrinoid necrosis are given double weight. The sum of the six components yields a total AI score of 0 to 24 . Likewise, a chronicity index (CI) of 0 to 12 is obtained from the sum of glomerulosclerosis, fibrous crescents, tubular atrophy, and interstitial fibrosis, each given 0 to $3+$. A combination of $\mathrm{AI}(>7)$ and $\mathrm{CI}(>3)$ predicts a poor long term outcome in lupus nephritis. ${ }^{1,4,17,27,28}$

In addition to the class of lupus nephritis, the biopsy report should include percentage of glomeruli with fibrinoid necrosis/ crescents, percentage of glomeruli with chronic lesions, documentation and grading of the extent, severity and type of tubulointerstitial and vascular disease and AI and CI scores. Finally, biopsy findings must be put into the context of the patient by the treating nephrologist. ${ }^{17}$

\section{Clinicopathological Correlation in Lupus Nephritis}

There is usually good correlation between degree of glomerular involvement and clinical renal involvement in lupus (table 2). ${ }^{1}$

Table 2: Clinicopathological correlation in lupus nephritis. ${ }^{1,4,28,29}$

\section{ISN/RPS Class of LN Clinical \& serological features}

Class I

Class II

Class III A or $\mathrm{A} / \mathrm{C}$

Class III C

Class IV A

Class V

Class VI
No evidence of clinical renal disease; excellent renal prognosis

Inactive urinary sediment, hypertension is infrequent, GFR preserved, proteinuria is usually $<1 \mathrm{~g}$ /day; excellent renal prognosis

Microhematuria, hypertension, proteinuria, active lupus serologies; proteinuria often $>1 \mathrm{~g}$ /day; one-fourth to one-third have nephrotic syndrome; up to $25 \%$ have elevated serum creatinine at renal biopsy.

Hypertension and reduced renal function, inactive urinary sediment

High serologic activity, active urinary sediment, hypertension, reduced GFR and heavy proteinuria, almost $50 \%$ have nephrotic syndrome.

Typically have proteinuria and nephrotic syndrome; hypertension and renal dysfunction can be present; sometimes can present just as idiopathic nephrotic syndrome; increased risk of thrombotic complications.

Advanced sclerotic LN or end stage LN, usually "burnt-out" serologically inactive disease; many patients nevertheless have persistent microhematuria and some proteinuria. 
Sometimes, lupus patients with class I or class II biopsies develop lupus podocytopathy and present with sudden onset of nephrotic syndrome. ${ }^{30}$ Crescentic lupus nephritis behaves more like pauci immune GN and presents with rapidly progressive renal failure. ${ }^{29}$ Patients with mixed class V and IV have clinical features that reflect both components. ${ }^{17}$

\section{Natural history and prognosis of lupus nephritis}

The natural history of LN is changing with the availability of new immunosuppressives, their judicious use and improvement in supportive care. Relapses depend not only on the disease severity but also on the intensity and duration of immunosuppression. ${ }^{1,4}$ There is bimodal pattern of mortality in lupus, early mortality is due to disease activity and infections while almost half of the later deaths are due to cardiovascular causes. Overall survival in patients with SLE in the modern era is approximately $95 \%$ at 5 years and $92 \%$ at 10 years after diagnosis. The presence of lupus nephritis (LN) significantly reduces survival to approximately $88 \%$ at 10 years. 12 ESRD now affects $8 \%$ to $15 \%$ of patients with LN.,4 The various factors affecting outcome in LN have been enumerated in table 3.

Table 3: Factors associated with outcomes in $\mathrm{LN}^{1,2,4,6,7,24,28,29,31-34}$

Epidemiologic and demographic factors

African Americans and Hispanic Americans do worse; Southeast Asians have more severe disease than other Asians or Caucasians; male gender, younger age and lower socioeconomic status are associated with worse renal outcomes.

$\begin{array}{ll}\text { Clinical and laboratory } & \\ \text { outcome predictors } & \begin{array}{l}\text { Higher serum creatinine or greater proteinuria, hypertension, severe anemia \& } \\ \text { thrombocytopenia at baseline are predictors of poor renal outcome; reduced }\end{array} \\ & \begin{array}{l}\text { complement with elevated anti-dsDNA levels correlate with active renal involvement, but } \\ \text { not with long-term prognosis. }\end{array}\end{array}$

Clinical management predictors

Delay between onset of nephritis and renal biopsy, delay to start therapy, and faster rate of decline of GFR predict worse prognosis; failure to achieve remission, renal (nephritic) flares and complications of treatment negatively affect outcome; early response to therapy at month is the predictor of good long-term outcome.

Histological features

Patients with class I and II lesions generally have excellent prognosis. Patients with severe proliferative $\mathrm{LN}$ with extensive necrosis and crescents, those with combined AI $>7$ plus CI $>3$ are more likely to have progressive renal failure; extensive tubular atrophy and interstitial fibrosis are independent negative prognostic markers. Membranous LN has better outcome in the short term; in long term, those with persistent nephrotic syndrome have worse outcome. In repeat biopsy at 6 months, ongoing inflammation, cellular crescents and persistent immune deposits are strong predictors of doubling of serum creatinine or progression to ESRD.

\section{Monitoring Clinical Disease}

In lupus, it is important to be able to predict systemic and renal relapses and prevent them through judicious use of immunosuppressives. The reasons for monitoring LN include - detecting renal remission \& renal flares, differentiating between flare and chronic damage, assessing likely duration of immunosuppression, assess extra-renal SLE and detect treatment-related toxicity. ${ }^{1,7,34}$ The monitoring parameters and the rationale are summarized in table 4. 
Table 4: Methods of monitoring in LN. ${ }^{7,12,35,36}$

\begin{tabular}{|c|c|}
\hline Monitoring parameters & Reason \\
\hline Urinalysis and microscopy & $\begin{array}{l}\text { To detect active sediments in active LN, leucocytes in interstitial nephritis or } \\
\text { urinary tract infection, malignant cells in bladder malignancy }\end{array}$ \\
\hline Serum creatinine / eGFR & Renal function \\
\hline Proteinuria & $\begin{array}{l}\text { An increase in proteinuria from }<1 \mathrm{~g} / \text { day to over this amount and from non } \\
\text { nephrotic to nephrotic levels indicates either increased activity or a change in } \\
\text { renal histologic class }\end{array}$ \\
\hline Autoimmune serology & $\begin{array}{l}\text { Serum levels of anti-ds DNA and anti-C1q antibodies typically increase and } \\
\text { complement levels typically decrease as the clinical activity of SLE increases. } \\
\text { Combination anti-C1q and anti-dsDNA antibodies better predicts renal prognosis } \\
\text { in LN. }\end{array}$ \\
\hline Repeat renal biopsy & $\begin{array}{l}\text { For change in histologic class, AI, CI and changes of antiphospholipid syndrome } \\
\text { nephropathy (APSN). Indications for repeat biopsy are: improved renal disease } \\
\text { but persistence of proteinuria, persistent or relapsing nephrotic syndrome and } \\
\text { increase in plasma creatinine by at least } 50 \% \text {. }\end{array}$ \\
\hline Novel urine markers & $\begin{array}{l}\text { Urinary monocyte chemoattractant protein-1(MCP-1), TNF-related weak inducer } \\
\text { of apoptosis (TWEAK), lipocalin-2, proteomics, urine C } 3 \mathrm{~d} \text { - have been variously } \\
\text { found to reflect activity of LN }\end{array}$ \\
\hline
\end{tabular}

In clinical practice, monitoring involves blood pressure, urinalysis, proteinuria, serum creatinine / eGFR, C3/C4 levels, anti-dsDNA levels, antiphospholipid antibodies, infection and cardiovascular risks and issues of vaccination, bone health and compliance. ${ }^{7,12}$

\section{Pregnancy and SLE}

The two important issues in pregnancy are:

1. How pregnancy will affect lupus - Lupus flares in $>$ $50 \%$ of the pregnancies. If conception occurs during active renal disease, flare rate is $48-62 \%$, compared to $7.4-32 \%$ when it occurs during remission. Patients with quiescent lupus at the time of pregnancy are less likely to experience flares. ${ }^{21,23,37,38}$

2. How lupus will affect the pregnancy - pregnancies in lupus patients are associated with an increased risk of adverse maternal and fetal outcomes. A history of lupus nephritis before conception predicts adverse maternal outcome, flare during pregnancy and low birth weight baby while flare during pregnancy predicts adverse fetal outcomes. ${ }^{10}$ The maternal risks include worsening of renal function, hypertension, proteinuria and preeclampsia \& eclampsia. ${ }^{10,21,23,37,38}$ Fetal outcomes include increased risk of miscarriage or stillbirth, small for gestational age, intra-uterine growth retardation and preterm delivery. ${ }^{37.38}$ Raised serum creatinine, antiphospholipid antibodies and anti-SSA/Ro and anti-
$\mathrm{SSB} / \mathrm{La}$ antibodies affect fetal outcome. The rate of fetal loss in all SLE patients is $20 \%$ to $40 \%$ and may approach $50 \%$ in some series. In a study, fetal death occurred in $38 \%$ to $59 \%$ of all pregnant SLE patients with APL antibodies compared with $16 \%$ to $20 \%$ of those without these antibodies. ${ }^{1,21,38,39}$ Children born to mothers positive for anti-SSA/Ro and anti-SSB/ $\mathrm{La}$ antibodies are at risk for congenital heart block. It is advisable to delay pregnancy until the disease is in remission for at least 6 months. ${ }^{10}$

\section{Antiphospholipid Antibody Syndrome (APS)}

Antiphospholipid antibodies (APL) are present in approximately one quarter of lupus pregnancies. ${ }^{39}$ Lupusassociated APS is actually more common than primary APS.21 The two major clinical manifestations of APS are thrombosis and obstetric complications. ${ }^{21,22}$ APS is diagnosed if at least one clinical criteria is present with persistence of high titers of APL autoantibodies for $>12$ weeks. $^{21,39}$ LN with APS is accompanied by more hypertension, azotemia, interstitial fibrosis and worse outcomes than it without APS.22 The diagnosis of antiphospholipid antibody syndrome nephropathy (APSN) is made if thrombotic microangiopathy involving both arterioles and glomerular capillaries is present and/ or one or more of fibrous intimal hyperplasia involving organized thrombi, fibrous and/or fibrocellular occlusions of arteries and arterioles, focal cortical atrophy or tubular 
thyroidisation (large zones of atrophic tubules containing eosinophilic casts). ${ }^{7}$

\section{Management}

SLE requires a multidisciplinary management. ${ }^{7}$ Only four drugs are so far approved by United States Food and Drug Administration (FDA) for lupus, they being aspirin, glucocorticoids, hydroxychloroquine and belimumab. ${ }^{13}$ The aims in the care of patients with lupus nephritis go beyond drugs and beyond immunosuppression. "Right drug, right dose, right patient, right time", this aphorism applies in lupus care since treatment options are many and the disease has chronic and fluctuating course. ${ }^{7}$ The aims in lupus care can be summed up as: .,40-42 $^{7}$

- $\quad$ Avoid unnecessary immunosuppression.

- Obtain early complete remission, maintain remission and prevent flares.

- $\quad$ Control proteinuria, blood pressure and manage vascular risk factors.

- Identify and treat APS.

- $\quad$ Address pregnancy issues.

- $\quad$ Assess infection risk, assure bone health \& adjunctive therapies.

- $\quad$ Minimize iatrogenic toxicity \& morbidity.

- $\quad$ Ensure adherence \& reduce overall mortality.

Use of ISN/RPS classification serves as a guide to initial therapy. ${ }^{1,4,6,9,11,12,17,24,40}$ Patients with class I and II biopsies need no therapy directed at kidney. Sometimes, they have nephrotic syndrome due to concomitant lupus podocytopathy; these patients are treated with steroids or calcineurin inhibitors similar to patients with minimal change disease (MCD). If there is increased proteinuria and active urinary sediments, repeat renal biopsy is required. ${ }^{1,4,6,8,13,24,30}$

Patients with ISN/RPS class III, IV and V generally need specific therapies targeting kidney. It consists of combinations of steroids, immunosuppressives and newer immunotherapies, depending on the histopathology, patient profile, response to initial therapy and availability and affordability of therapy. ${ }^{1,4,6-9,11,12,24,40}$ Efficacy of therapy has to be carefully weighed against toxicity. ${ }^{1}$

Treatment of LN has evolved over time. It is the current standard of care to divide the treatment of patients with active proliferative $\mathrm{LN}$ into an initial or induction phase and a maintenance phase. Induction phase consists of aggressive therapies to control acute life- or organ- threatening disease. The maintenance phase focuses on the long-term management of chronic, indolent disease along with maintenance of cardiovascular health, protection from the side effects of therapy and prevention of flares. ${ }^{1,4,6,8,12,24,40}$ The new paradigm in the management is to use low dose steroid, avoid high dose and prolonged use of cyclophosphamide and in the future drastically reduce or eliminate the requirement for long-term steroids and if possible avoid the need for any maintenance therapies. ${ }^{5,6,8,11-14,24}$

ISN/RPS class III patients with only few mild proliferative lesions and no necrotizing features or crescents have a good prognosis and often respond to a short course of high-dose corticosteroid therapy or a brief course of other immunosuppressive agents. Patients with greater number of affected glomeruli and those with necrosis and crescents usually require more vigorous therapy similar to patients with class IV. ${ }^{1,8,12,24}$

For induction therapy of class III and class IV LN, mycophenolate mofetil (MMF) (2-3 gm/day oral) or iv cyclophosphamide (IVC) along with glucocorticoids is recommended. MMF has similar efficacy in all races. ${ }^{1,4,6,8,9,12,24,25,40,42-52}$ Asians might require lower doses of MMF. MMF may be an initial choice in patients who are African American or Hispanic. ${ }^{12}$ Opinions are favoring MMF as first-line induction and maintenance treatment therapy compared to cyclophosphamide (CYC) for LN.53 There are two regimes of IVC: 1) low-dose CYC "EuroLupus regime" (500 mg iv every 2 weeks for a total of 6 doses), followed by maintenance therapy with daily oral azathioprine (AZA) or daily oral MMF, and 2) high-dose CYC (500-1,000 mg/m2 iv monthly for 6 doses), followed by maintenance treatment with $\mathrm{MMF}$ or AZA. ${ }^{12,24,43-47}$ The lower "Euro-Lupus" regime of IVC is recommended for white patients with clinically less severe disease. ${ }^{12,24}$ Especially for severe disease, pulse iv methylprednisolone (500-1,000 mg daily for 3 doses) followed by daily oral prednisolone $(0.5-1 \mathrm{mg} / \mathrm{kg} / \mathrm{day})$, the dose then reduced stepwise to approximately $10 \mathrm{mg}$ /day by $3-6$ months is recommended. ${ }^{12,24}$ After initiation of induction treatment, major changes in treatment, other than change of steroid doses, should not be made till 6 months of therapy unless there is clear evidence of worsening at 3 months $(\geq 50 \%$ worsening of proteinuria or serum creatinine). A repeat kidney biopsy may be considered at this point. ${ }^{12,24}$

The dose of IVC is reduced by $20 \%$ or $30 \%$ in patients with creatinine clearance $<25-50$ and $10-25 \mathrm{ml} / \mathrm{min}$, respectively and adjusted for some removal by hemodialysis in ESRD patients. The dose of IVC should be adjusted to keep the day 10-14 leukocyte count nadir $\geq 3000 / \mathrm{ml}$. 
Mercaptoethane sulfonate (MESNA) therapy reduces bladder complications of CYC. ${ }^{1,24}$ Oral CYC $1.0-1.5 \mathrm{mg} /$ $\mathrm{kg} / \mathrm{d}$ (maximum dose $150 \mathrm{mg} / \mathrm{d}$ ) for $2-4$ months is effective but has more adverse effects compared to IVC. ${ }^{24,54}$ With oral CYC, white cell counts should be monitored weekly and $\mathrm{CYC}$ dose should be adjusted to keep leucocytes $\geq 3000 / \mathrm{ml}$. To minimize bladder toxicity oral CYC should be taken in the morning with extra fluid. ${ }^{24}$

MMF is preferred when fertility is a concern, since six months of high-dose IVC is associated with approximately $10 \%$ sustained infertility in young women, and higher rates in older women. ${ }^{6,12,24,40}$ Leuprolide and testosterone are not established. Ovarian tissue cryopreservation is an expensive option; sperm banking should be offered. Since MMF is teratogenic, it should be stopped for at least 6 weeks before attempting pregnancy. CNIs have been tested in small trials; they give good results especially with persistent heavy proteinuria. CNIs have also been part of multitarget therapy. ${ }^{6,8,24,40,55}$

For induction treatment of patients with Class IV or IV/V plus cellular crescents, either CYC or MMF along with iv pulses of methylprednisolone followed by prednisolone $1 \mathrm{mg} / \mathrm{kg} /$ day orally is recommended. ${ }^{12}$ Experts favor high-dose IVC protocol for LN with cellular crescents. ${ }^{12}$ however, a subset of patients in the ALMS trial did have severe $\mathrm{LN}$ and responded to MMF. ${ }^{24}$

If nephritis is worsening at 3 months or patients fail to respond after 6 months of treatment with steroid plus MMF or steroid plus CYC, a switch from either CYC to MMF, or from MMF to CYC, is recommended, with these changes accompanied by iv pulses of MP for 3 days. Combinations of MMF and calcineurin inhibitors and of rituximab and MMF might be considered for those who have failed the recommended induction therapies. ${ }^{12,24,55}$

Patients with pure class $\mathrm{V}$ LN and with nephrotic range proteinuria are started on prednisone $(0.5 \mathrm{mg} / \mathrm{kg} /$ day $)$ plus MMF 2-3 gm /day continued for 6 months. ${ }^{12,24}$ If improved with the initial therapy, MMF 1-2 gm/day or AZA $2 \mathrm{mg} /$ $\mathrm{kg}$ /day is kept for maintenance. If not improved with initial therapy, IVC $500-1000 \mathrm{mg} / \mathrm{m} 2$ monthly pulse for six months plus methylprednisolone pulse followed by prednisolone 0.5 to $1 \mathrm{mg} / \mathrm{kg} /$ day is used.12,56,57 Alternate therapies include cyclosporine $4-6 \mathrm{mg} / \mathrm{kg} / \mathrm{d}$ for $4-6$ months or AZA 1-2 mg/kg/d for 6 months. Tacrolimus can substitute cyclosporine. ${ }^{57,58}$ For asymptomatic membranous LN with subnephrotic range proteinuria with preserved GFR, options include prednisolone daily for 2-6 months or low dose cyclosporine or therapy with ACE inhibitors (ACEi) or angiotensin receptor blockers (ARB) and statins; in all cases, patients need close follow. ${ }^{4}$
After the initial phase, maintenance therapy consists of either AZA or MMF \pm low dose glucocorticoids. 1,4,6,8,12,24,40,51,59,60 Again, patient-specific factors dictate the choice between MMF or AZA. ${ }^{24}$ Azathioprine is given at $1.5-2.5 \mathrm{mg} / \mathrm{kg} / \mathrm{d}$, MMF $1-2 \mathrm{~g} / \mathrm{d}$ in divided doses and low-dose oral corticosteroids ( $\leq 10 \mathrm{mg} / \mathrm{d}$ prednisolone). CNIs may substitute MMF or azathioprine in patients not tolerating the latter. ${ }^{24}$ To date, there are no adequate data to guide how rapidly AZA or MMF can be tapered or withdrawn. ${ }^{7,24}$ After complete remission is achieved, maintenance therapy is continued for at least 1 year before considering tapering of immunosuppression, though there is no agreement on definitions of terms such as remission, flare, and response and there is no specific recommendation for tapering of immunosuppressives. It is often possible to stop treatment entirely in many patients after 5 years or more, when the disease process has apparently "burned out." Stable GFR, lack of proteinuria, and normal immunologic tests predict successful discontinuation of immunosuppressives. ${ }^{61}$ However, at 10 -year follow-up of the Euro-Lupus study, $75 \%$ patients were still receiving one of those therapies. ${ }^{46}$ While tapering, if kidney function deteriorates and/or proteinuria worsens, treatment should be increased to the previous level of immunosuppression that controlled the LN. If complete remission has not been achieved after 12 months of maintenance therapy, change in therapy should be guided by repeat kidney biopsy. ${ }^{24}$

\section{Treatment of LN in Pregnancy}

Management begins with preconception counseling. In patients with history of LN but the disease quiescent currently, no medications are necessary. For mild systemic activity hydroxychloroquine is given. For clinically active nephritis treatment options are prednisolone, and if necessary AZA ( $\leq 2 \mathrm{mg} / \mathrm{kg}$ in pregnant); fluorinated glucocorticoids should be avoided in pregnancy. CNIs are other options. Rituximab is not recommended; MMF, CYC and methotrexate are teratogenic. If patients have persistently active nephritis with documented or suspected class III or IV with crescents, delivery after 28 weeks should be considered. ${ }^{1,12,38}$ For patients with pregnancy and APS with past thrombosis, anticoagulation seems to improve both maternal and fetal outcomes. ${ }^{21}$ Women with a history of APS and arterial thrombotic events should be advised against pregnancy due to high risks of not only pregnancy loss, but also stroke and maternal morbidity and mortality. ${ }^{12,38}$

\section{Newer therapeutic options for lupus}

Current therapy for lupus is far less than optimal. It is not universally effective, side effects are many; renal response 
rate for class III and IV LN with any of the initial therapies so far in use is only about $60 \%$ at 6-12 months. Long term therapy is needed and there is risk of disease flare; relapse rate for $\mathrm{LN}$ ranges from $35-60 \%{ }^{6,8} \mathrm{New}$ therapeutic options and immunotherapies are being explored. ${ }^{62}$ The major goal of future therapies are: ${ }^{13,14,40}$

- To drastically reduce or eliminate the requirement for long-term steroids.

- To develop biologic therapies and small-molecule drugs influencing particular immune cells (e.g. B cells) \& molecules (e.g. costimulatory molecules, cytokines) so that disease control is achieved with lower toxicity and without wide-ranging suppression of the immune system.

The options include: $:^{1,3,5,6,11-14,40,62}$

- $\quad$ B cell targeted therapy

o Rituximab - a chimeric anti-CD20 monoclonal antibody.

o Ocrelizumab - a humanized anti CD 20 monoclonal antibody.

o Epratuzumab - humanized anti-CD22 monoclonal antibody.

o Belimumab: inhibitor of the cytokine B-lymphocyte stimulator (BLyS) or B cell activating factor (BAFF). FDA approved belimumab for use in seropositive patients of SLE who have active disease in spite of prior therapies.

o Atacicept - TACI immune globulin.

- Tocilizumab - Monoclonal antibody against the interleukin-6 receptor

- $\quad$ Eculizumab- antibody inhibiting complement C5

- Blockade of interleukin- 17 \& interleukin-23

- $\quad$ Abetimus - induces tolerance, prevents anti-DNA antibody formation and prevents flares.

- $\quad$ Abatacept, belatacept - block interaction between T and $\mathrm{B}$ cells ie costimulation blockade.

- Hydroxyxhloroquine - inhibition of toll like receptor of APCs.

- Blockade of type I interferon

- $\quad$ Plasma exchange- for patients with severe pulmonary hemorrhage, TTP-like syndrome, anticardiolipin antibodies and a clotting episode who cannot be anticoagulated.
More generalized immune strategies ie non specific immunotherapy

o Intravenous gamma globulin (IVIg)

o Total lymphoid irradiation and immunoablation.

- $\quad$ Regulating end-organ responses with targeted drug delivery technologies, immunoliposomal systems to deliver drugs to the glomeruli.

Gene therapy to regulate local inflammation in the mesangium.

Additional points in care of lupus patients

- Some experts recommend low-dose aspirin and hydroxychloroquine for asymptomatic patients with APL antibodies. If there is clinical thrombotic event, chronic anticoagulation is advised. ${ }^{1,21,22}$

- Use of hydroxychloroquine in SLE leads to lower lupus flare rates, lower renal damage, reduced clotting events, is negatively associated with hypertension and infection, and has a better survival rate. Hydroxychloroquine is recommended in all LN patients unless contraindicated. ${ }^{12}$

- The risk of heart attack in young women with SLE is 50 times higher than that of healthy women; even older women with SLE have 2.5 to 4 times higher risk of myocardial infarction. ${ }^{18}$ Atherosclerotic risk is reduced with tight control of blood pressure $(\leq 130 / 80 \mathrm{mmHg})$, use of statins and suppression of active disease. ACR recommends statin for patients with LDL cholesterol $>100 \mathrm{mg} / \mathrm{dl}^{12,18}$

- Women with SLE have five times higher fracture rates than normal women do. The strategies for bone protection include: minimizing use of steroids, supplement vitamin D and calcium, bisphosphonate use in high risk patients, regular assessment of bone mineral density every 2-3 years and management of secondary hyperparathyroidism. ${ }^{4,6,7,24}$

- $\mathrm{ACEis}$ or $\mathrm{ARBs}$ are antiproteinuric and renoprotective. In LUMINA lupus cohort use of ACEi was found to delay the occurrence of nephritis. ${ }^{1,4,7,63}$

- Blood pressure should be controlled to $\leq 130 / 80$ $\mathrm{mmHg}$ in general and $<125 / 75 \mathrm{mmHg}$ in patients with proteinuria $>1 \mathrm{~g} /$ day, $\mathrm{ACEi} / \mathrm{ARB}$ being the agent of choice. ${ }^{7,12}$ In lupus patients with resistant hypertension and APS, renal artery stenosis should be suspected. ${ }^{7}$

Pneumococcal and influenza vaccines are recommended. Live vaccines can trigger lupus flares and 
are contraindicated while on immunosuppressives or high doses of steroid. ${ }^{12,25}$

\section{Dialysis and Transplantation}

Lupus represents $1 \%$ to $2 \%$ of all patients with ESRD. ${ }^{1,4}$ Many patients have inactive burnt-out disease by the time they reach ESRD, clinical lupus activity wanes with prolonged time on dialysis. ${ }^{1,4,64}$ In the early months of dialysis, lupus patients have increased mortality because of infectious complications of immunosuppressive therapy, however in the longer term their survival on dialysis, is comparable to that of other primary renal diseases. ${ }^{1,4,64}$ Patients with APS benefit from anticoagulation to prevent arteriovenous fistula or graft clotting. ${ }^{4}$

Outcomes in SLE patients undergoing transplantation are, in general, similar to those of patients with other diseases. ${ }^{64}$ The rate of recurrent nephritis in the allograft has been low-less than 4\%. Most of the recurrences seen in surveillance biopsies are subclinical, mild mesangial. ${ }^{1,64}$ Some additional considerations during transplantation of lupus patients include: $1,4,21,64$

- Patients with active SLE may be kept in dialysis for 3 to 12 months before taking up for transplant to allow clinical and serologic disease activity to become quiescent.

- Cross matching of donors with lupus patients may be difficult because the sera may contain anti lymphocyte autoantibodies, rendering a false-positive "cross match."

- For patients who have clinically inactive but serologically active lupus, starting transplant immunosuppressives several weeks to a month before transplantation is an option.

- Thrombosis after transplantation may be a problem in patients with APS. Anticoagulation therapy during the post transplant period may benefit these patients.

Conflict of interest: The authors declare that they have no conflict of interests.

\section{References}

1. Appel GB, Radhakrishnan J, D'Agati VD. Secondary glomerular diseases. In: Taal MW, Chertow GM, Marsden PA, Skorecki K, Yu ASL, Brenner BM, editors. Brenner \& Rector's The Kidney. Philadelphia: Elsevier Saunders. 2012; 1192-1277

2. Ayodele OE, Okpechi IG, Swanepoel CR. Predictors of poor renal outcome in patients with biopsy-proven lupus nephritis. Nephrology 2010;15: 482-490
3. Bagavant H, Kalantarinia K, Scindia Y, Deshmukh U. Novel therapeutic approaches to lupus glomerulonephritis: translating animal models to clinical practice. Am J Kidney Dis 2011; 57(3):498-507

4. Appel GB, Jayne D. Lupus nephritis. In: Floege J, Johnson RJ, Feehally J, editors. Comprehensive clinical nephrology. St Louis Missouri: Elsevier Saunders. 2010; 308-321

5. Rahman A, Isenberg DA. Systemic lupus erythematosus. N Engl J Med 2008; 358:929-939

6. Bomback AS, Appel GB. Update on the treatment of lupus nephritis. J Am Soc Nephrol 2010; 21:2028-2033.

7. Masood S, Jayne D, Karim Y. Beyond immunosuppression - challenges in the clinical management of lupus nephritis. Lupus 2009; 18:106115.

8. Waldman W, Appel GB. Update in the treatment of lupus nephritis. Kidney Int 2006; 70: 1403-12.

9. Bertsias G, Ioannidis J P A, Boletis J et al. EULAR recommendations for the management of systemic lupus erythematosus. Report of a task force of the EULAR standing committee for international clinical studies including therapeutics. Ann Rheum Dis 2008; 67:195-205.

10. Kwok L-W, Tam L-S, Zhu TY, Leung Y-Y, Li EK. Predictors of maternal and fetal outcomes in pregnancies of patients with systemic lupus erythematosus. Lupus 2011; 20:829-836.

11. Bertsias GK, Salmon JE, Boumpas DT. Therapeutic opportunities in systemic lupus erythematosus: state of the art and prospects for the new decade. Ann Rheum Dis 2010; 69:1603-1611.

12. Hahn BH, MC Mahon MA, Wilkonsin A et al. American College of Rheumatology guidelines for screening, treatment, and management of lupus nephritis. Arthritis Care \& Research 2012; 64(6): 797-808.

13. Bhat P, Radhakrishnan J. B lymphocytes and lupus nephritis: new insights into pathogenesis and targeted therapies. Kidney Int 2008; 73: 261-268

14. Tsokos GC. Systemic lupus erythematosus. N Engl J Med 2011;365: 2110-21.

15. Vlag J, Berden JHM. Lupus nephritis: role of antinucleosome autoantibodies. Semin nephrol 2011; 31(4):376-389.

16. Cervera R, Khamashta MA, Font J, et al. Systemic lupus erythematosus: clinical and immunologic patterns of disease expression in a cohort of 1,000 patients. 
The European Working Party on Systemic Lupus Erythematosus. Medicine (Baltimore) 1993; 72:11324.

17. Weening JJ, D'Agati VD, Schwartz MM, et al. The classification of glomerulonephritis in systemic lupus nephritis revisited. Kidney Int 2004; 65:521-530.

18. Urowitz MB, Gladman D, Ibanez D, et al. Clinical manifestations and coronary artery disease risk factors at diagnosis of systemic lupus erythematosus: data from international inception cohort. Lupus 2007;16:7315 .

19. Valdivielso P, Gómez-Doblas JJ, Macias M, et al. Lupus-associated endothelial dysfunction, disease activity and arteriosclerosis. Clin Exp Rheumatol 2008; 26: 827-33.

20. Hochberg MC. Updating the American College of Rheumatology (ACR) revised criteria for the classification of systemic lupus erythematosus, Arthritis Rheum 1997; 40(9):1725.

21. Hill GS, Nochy D. Antiphospholipid syndrome in systemic lupus erythematosus. J Am Soc Nephrol 2007; 18:2461-2464.

22. Giannakopoulos B, Krilis SA. The pathogenesis of the antiphospholipid syndrome. N Engl J Med 2013; 368:1033-44.

23. Imbasciati E, Tincani A, Gregorini G, et al. Pregnancy in women with pre-existing lupus nephritis: predictors of fetal and maternal outcome. Nephrol Dial Transplant $2009 ; 24: 519-525$.

24. KDIGO clinical practice guideline for glomerulonephritis, chapter 12: lupus nephritis. Kidney Int Supple 2012; 2:221-232.

25. Nasr SH, D'Agati VD, Park HR, et al. Necrotizing and crescentic lupus nephritis with antineutrophil cytoplasmic antibody seropositivity. Clin J Am Soc Nephrol 2008; 3:682-690.

26. Jennette JC, Iskander SS, Dalldorf FG. Pathologic differentiation between lupus and nonlupus membranous glomerulopathy. Kidney Int 1983; 24:377.

27. Schwartz MM. The Holy Grail: pathological indices in LN. Kidney Int 2000; 58:1354-1355.

28. Austin HA, Boumpas DT, Vaughan EM, et al. Predicting renal outcomes in severe lupus nephritis: contributions of clinical and histologic data. Kidney Int 1994; 43:544550 .

29. Yu F, Tan Y, Liu G, Wang S, Zou W, Zhao M. Clinicopathological characteristics and outcomes of patients with crescentic lupus nephritis. Kidney Int 2009; 76: 307-317.

30. Dube GK, Markowitz GS, Radhakrishnan J, et al. Minimal change disease in SLE. Clin Nephrol 2002; 57:120-126.

31. Faurschou M, Starklint H, Halberg P, Jacobsen SJ. Prognostic factors in lupus nephritis: diagnostic and therapeutic delay increases the risk of terminal renal failure. Rheumatology 2006; 33:1563-1569.

32. Houssiau FA, Vaconcelos C, D'Cruz D et al. Early response to immunosuppressive therapy predicts good renal outcomes in lupus nephritis: lessons from long term follow up of patients in the Euro- Lupus Nephritis trial. Arthritis Rheum 2004; 50:3934-3940.

33. Moroni G, Quaglini S, Maccario M, Banfi G, Ponticelli C. "Nephritic flares" are predictors of bad long-term renal outcome in lupus nephritis. Kidney Int 1996; 50: 2047-2053.

34. Korbet SM, Lewis EJ; for the Collaborative Study Group. Complete remission in severe lupus nephritis: assessing the rate of loss in proteinuria. Nephrol Dial Transplant 2012; 27:2813-2819.

35. Fernando MM, Isenberg DA. How to monitor SLE in routine clinical practice. Ann Rheum Dis 2005; 64: 524-527.

36. Rovin B, Zhang X. Biomarkers for lupus nephritis: the quest continues. Clin J Am Soc Nephrol 2009; 4:18581865 .

37. Stanhope TJ, White WM, Moder KG, Smyth A, Garovic VD. Obstetric nephrology: lupus and lupus nephritis in pregnancy. Clin J Am Soc Nephrol 2012; 7: 2089-2099.

38. Smyth A, Oliveira GH, Lahr BD, Bailey KR, Norby SM, Garovic VD: A systematic review and meta-analysis of pregnancy outcomes in patients with systemic lupus erythematosus and lupus nephritis. Clin J Am Soc Nephrol 2010; 5:2060-2068.

39. Miyakis S, Lockshin MD, Atsumi T et al. International consensus statement on an update of the classification criteria for definite antiphospholipid syndrome (APS). J Thromb Haemost 2006; 4: 295-306.

40. Ponticelli C, Glassock RJ, Gabriella M. Induction and maintenance therapy in proliferative lupus nephritis. JNephrol 2010; 23(01):9-16.

41. Chen YE, Korbet SM, Katz RS et al, Collaborative Study Group. Value of a complete or partial remission in severe lupus nephritis. Clin J Am Soc Nephrol 2008; $3: 46-53$ 
42. Felson DT, Anderson J. Evidence for the superiority of immunosuppressive drugs and prednisone over prednisone alone in lupus nephritis: results of a pooled analysis. N Engl J Med 1984; 311:1528-1533.

43. Austin HA 3rd, Klippel JH, Balow JE, et al. Therapy of lupus nephritis: controlled trial of prednisone and cytotoxic drugs. N Engl J Med 1986; 314:614-619.

44. Boumpas DT, Austin HA, Vaughn EM, et al. Controlled trial of pulse methylprednisolone versus two regimens of cyclophosphamide in severe lupus nephritis. Lancet 1992; 340:741-745.

45. Houssiau FA, Vasconcelos C, D’Cruz D, et al. Immunosuppressive therapy in lupus nephritis: The Euro-Lupus Nephritis Trial, a randomized trial of lowdose versus high-dose intravenous cyclophosphamide. Arthritis Rheum 2002; 46:2121-2131.

46. Houssiau FA, Vasconcelos C, D'Cruz D, et al. The 10year follow-up data of the Euro-Lupus Nephritis Trial comparing low-dose versus high-dose intravenous cyclophosphamide. Ann Rheum Dis 2010; 69:61-64.

47. Contreras G, Pardo V, Leclercq B, et al. Sequential therapies for proliferative lupus nephritis. N Engl J Med 2004; 350:971-980.

48. Walsh M, James M, Jayne D, et al. Mycophenolate mofetil for induction therapy of lupus nephritis: A systematic review and meta-analysis. Clin J Am Soc Nephrol 2007; 2:968-75.

49. Chan TM, Li FK, Tang CS, et al. Efficacy of mycophenolate mofetil in patients with diffuse proliferative lupus nephritis. Hong Kong- Guangzhou Nephrology Study Group. N Engl J Med 2000; 343: 1156-62.

50. GinzlerEM, DooleyMA,AranowC, etal.Mycophenolate mofetil or intravenous cyclophosphamide for lupus nephritis. N Engl J Med 2005; 353:2219-28.

51. Chan TM, Tse KC, Tang CS, et al. Long-term study of mycophenolate mofetil as continuous induction and maintenance treatment for diffuse proliferative lupus nephritis. J Am Soc Nephrol 2005; 16:1076-84.

52. Appel GB, Contreras G, Dooley MA, et al. Aspreva Lupus Management Study Group, Mycophenolate mofetil versus cyclophosphamide for induction treatment of lupus nephritis. J Am Soc Nephrol 2009; 20: 1103-12.

53. Hogan J, Schwenk MH, Radhakrishnan J. Should mycophenolate mofetil replaces cyclophosphamide as first-line therapy for severe lupus nephritis? Kidney Int 2012; 82:1256-1260
54. McKinley A, Park E, Spetie D, et al. Oral cyclophosphamide for lupus glomerulonephritis: an underused therapeutic option. Clin J Am Soc Nephrol 2009; 4:1754-1760.

55. Bao H, Liu ZH, Xie HL, et al. Successful treatment of class V+IV lupus nephritis with multitarget therapy. J Am Soc Nephrol 2008; 19: 2001-2010.

56. Radhakrishnan J, Moutzouris DA, Ginzler EM, Solomons N, Siempos II, Appel GB. Mycophenolate mofetil and intravenous cyclophosphamide are similar as induction therapy for class $\mathrm{V}$ lupus nephritis. Kidney Int 2010; 77(2):152-60.

57. Austin HA 3rd, Illei GG, Braun MJ, Balow JE. Randomized, controlled trial of prednisone, cyclophosphamide, and cyclosporine in lupus membranous nephropathy. J Am Soc Nephrol 2009; 20:901-911.

58. Szeto CC, Kwan BC, Lai FM, et al. Tacrolimus for the treatment of systemic lupus erythematosus with pure class V nephritis. Rheumatology (Oxford) 2008; 47:1678-1681.

59. Houssiau FA, D'Cruz D, Sangle $S$ et al., for the MAINTAIN Nephritis Trial Group. Azathioprine versus mycophenolate mofetil for long-term immunosuppression in lupus nephritis: results from the MAINTAIN Nephritis Trial. Ann Rheum Dis 2010; 9(12):2083-2089.

60. Dooley MA, Jayne D, Ginzler EM et al. Mycophenolate versus azathioprine as maintenance therapy for lupus nephritis. N Engl J Med 2011; 365:1886-1895.

61. Moroni G, Gallelli B, Quaglini S, et al. Withdrawal of therapy in patients with proliferative lupus nephritis: Long-term follow-up. Nephrol Dial Transplant 2006; 21:1541-1548.

62. Karim MY, Pisoni C N, Khamashta MA. Update on immunotherapy for systemic lupus erythematosuswhat's hot and what's not! Rheumatology 2009; 48:332-341.

63. Durán-Barragán S, McGwin G, Jr Vilá LM, Reveille JD, Alarcón GS. Angiotensin-converting enzyme inhibitors delay the occurrence of renal involvement and are associated with a decreased risk of disease activity in patients with systemic lupus erythematosus results from LUMINA (LIX): a multiethnic US cohort. Rheumatology (Oxford) 2008; 47: 1093-1096.

64. Contreras G, Mattiazzi A, Guerra G. Recurrence of lupus nephritis after kidney transplantation. J Am Soc Nephrol 2010; 21: 1200-1207. 